\title{
ARTICLES
}

\section{Nonhydrostatic compression of elastically anisotropic polycrystals. I. Hydrostatic limits of 4:1 methanol-ethanol and paraffin oil}

\author{
J. W. Otto and J. K. Vassiliou \\ Department of Physics, Villanova University, Villanova, Pennsylvania 19085 \\ G. Frommeyer \\ Max-Planck-Institut für Eisenforschung, Max-Planck-Strasse 1, D-40237 Düsseldorf, Germany \\ (Received 6 February 1997; revised manuscript received 7 July 1997)
}

\begin{abstract}
The compression of a foil of $\mathrm{Cu}_{3} \mathrm{Au}$ in 4:1 methanol-ethanol and paraffin oil pressure-transmitting media has been studied by energy-dispersive x-ray diffraction in a diamond-anvil cell. Line shifts and line profiles are used to analyze elastic and plastic strains. The increasing viscosity of 4:1 methanol-ethanol causes departures from hydrostatic conditions starting around $7 \mathrm{GPa}$ even while the medium is in a liquid state. The glass transitions in the range 5-7 GPa for paraffin oil and 12-13 GPa for 4:1 methanol-ethanol cause a volume incompressibility and discontinuity followed by isotropic compression behavior. The hydrostatic limit of a liquid with respect to a polycrystalline sample may lie at pressures considerably below the pressure of solidification. For nonideal powders, even small departures from nonhydrostatic conditions of the pressure transmitting medium, often termed quasihydrostatic, can have a strong influence on the compression behavior.

[S0163-1829(98)01406-4]
\end{abstract}

\section{INTRODUCTION}

Pressure-transmitting media serve to transform a macroscopic nonisotropic stress field set up by a pressure device into hydrostatic conditions acting on a sample. As the shear strength of these media increases under pressure, nonhydrostatic conditions are expected to develop. In a polycrystalline elastically anisotropic sample, nonhydrostatic stresses may be set up at grain boundaries due to the inability to accommodate shape changes in neighboring grains. The sample behaves elastically when the nonhydrostatic stresses are below the yield stress of the sample. Once the yield strength of a sample is exceeded, it may react by grain fracture or by plastic deformation and work hardening in the case of brittle and ductile materials, respectively. Nonhydrostatic stresses below and above the elastic limit may have a strong effect on linear and/or volume strains. It is thus important particularly for purposes of pressure calibration studies to have tools for detecting elastic and plastic strains. With these tools, the hydrostatic limits of pressure media and the effect of nonhydrostatic stresses on the compression behavior can then be studied.

Using line shifts and line broadening of the fluorescence of ruby single crystals distributed in a sample chamber in a diamond-anvil cell, the limits of hydrostaticity of various pressure media ( $\mathrm{NaCl}$, alcohol mixtures, and oils) with respect to a strong brittle solid $\left(\mathrm{Al}_{2} \mathrm{O}_{3}\right)$ were determined. ${ }^{1}$ The uniaxial stresses acting on an elastically compressed powder can be quantified by measuring the line shifts of lattice spacings as a function of $h k l$ and applying anisotropic elasticity theory. ${ }^{2}$ The type and probability of lattice defects can be determined from line positions and line profiles. ${ }^{3}$ The energy-dispersive $\mathrm{x}$-ray diffraction method was demonstrated to be suitable for the analysis of plastic deformation in special cases ${ }^{4}$ and applied to study the compression behavior of a $\mathrm{Cu}_{3} \mathrm{Au}$ foil in a diamond-anvil cell with $\mathrm{NaCl}$ as the pressure medium. ${ }^{5}$ The transition from hydrostatic to nonhydrostatic elastic compression of this weak ductile material was not observed clearly in that study because it occurred at low pressures (around $1 \mathrm{GPa}$ ). The hydrostatic limit of $\mathrm{NaCl}$ with respect to polycrystalline $\mathrm{Cu}_{3} \mathrm{Au}$ thus lies at pressures considerably below the limit of $\mathrm{NaCl}$ determined from pressure gradients measured by ruby fluorescence (roughly $4 \mathrm{GPa}$ ). ${ }^{1}$ The plastic deformation of $\mathrm{Cu}_{3} \mathrm{Au}$ starting at around $2 \mathrm{GPa}$ in $\mathrm{NaCl}$ leads to dramatic deviations from an equation of state. ${ }^{5}$ It was therefore considered to be of interest to investigate the hydrostatic limits of the common liquid pressure media 4:1 methanol-ethanol and paraffin oil with respect to polycrystalline $\mathrm{Cu}_{3} \mathrm{Au}$ (part I) and to obtain further insight into the effects of nonhydrostatic compression. The hydrostatic pressure limits of 4:1 methanol-ethanol and of a 1:1 pentane-isopentane mixture have previously been regarded to lie at glass transitions around 10 and $7 \mathrm{GPa}$, respectively. ${ }^{1}$

In static high-pressure experiments, the uniaxial stress components can be increased relative to the hydrostatic stress at a given applied force by choosing pressure media of increasing shear strength. The limiting case is the direct compression of a material between two anvils without gasket or pressure medium (part II). This case is the low-strain rate, constant (low) temperature equivalent of the dynamic shock compression experiments and is therefore of importance for primary and secondary pressure calibration studies. In order to better understand the compression curves for samples showing both elastic and plastic behavior, special emphasis is placed on studying the compression behavior of solids previously deformed either at ambient conditions or by compression (part II). 


\section{METHODOLOGY}

\section{A. Experimental procedure}

The choice of sample and experimental parameters has been described in detail before. ${ }^{5}$ Briefly, a splat-quenched foil of ductile disordered $\mathrm{Cu}_{3} \mathrm{Au}$ is used because of its strong diffraction, good powder averaging, and sensitivity to shear stresses. A foil is the most extreme case of a nonideal powder (as far as the elastic properties are concerned). A MaoBell-type diamond-anvil cell ${ }^{6}$ was used as the pressure generating device.

The sample chamber consisted of a $150 \mu \mathrm{m}$ diameter hole in a spring steel gasket $70-80 \mu \mathrm{m}$ thick previously filled with the liquid pressure transmitting media. No special precautions were taken to dry the pressure transmitting media. From a foil of $\mathrm{Cu}_{3} \mathrm{Au} 20-50 \mu \mathrm{m}$ thick, a single flake (in the case of the experiment with 4:1 methanol-ethanol) or several flakes (in the case of paraffin oil) were carefully pried loose and dropped into the sample chamber. Care was taken to ensure that the flakes did not make contact with either gasket or diamonds, and that they were not tilted from the horizontal plane. In order to keep the foil floating in methanolethanol, pressure was immediately raised to $2.6 \mathrm{GPa}$. Pressure was measured from the fluorescence ${ }^{7,8}$ of a single grain of ruby 5-10 $\mu \mathrm{m}$ large attached to the piston diamond using vacuum grease. With the setup as described, the pressure determined from ruby under hydrostatic conditions is the confining pressure on the sample ${ }^{5}$ and it is labeled as such on all graphs. The diffraction angle for the experiment with methanol-ethanol was $2 \theta=10.668^{\circ}$. Exposure times were 2-3 $\mathrm{h}$ on pressure increase and $1.5 \mathrm{~h}$ on pressure release. Two experiments with an undeformed foil in paraffin oil were carried out. The first experiment at $2 \theta=10.852^{\circ}$ was stopped at $13.9 \mathrm{GPa}$ because the gasket hole had expanded strongly starting at $8.6 \mathrm{GPa}$. The second experiment up to 30 $\mathrm{GPa}$ and the same initial angle was interrupted twice (at 11.2 $\mathrm{GPa}$, the last spectrum saved was at $9.9 \mathrm{GPa}$; and at $20 \mathrm{GPa}$ ) by power failures necessitating recalibration of the detector and realignment of the diffraction setup. The experiment was continued after breaks of 7 and 10 days, respectively, with slightly different diffraction angles. It should be kept in mind that the sample spot investigated throughout the experiment may not have been the same because of the necessary realignment after the power failures.

The experiments were carried out at beamline F3 at Hamburger Synchrotron Strahlungslabor at Deutsches Elektronen Synchrotron with the setup described previously. ${ }^{9}$ Using a small primary beam $(40 \times 40 \mu \mathrm{m})$ and tight collimation of the diffracted beam in both the horizontal and vertical direction, the resolution function can be parametrized in the following way in the angular range used here:

$$
\frac{\Delta E_{\mathrm{FWHM}}}{E}=\sqrt{\frac{(0.154)^{2}}{E^{2}}+\frac{5.546 * 0.10 * \epsilon}{E}},
$$

where $\Delta E_{\mathrm{FWHM}}$ is the full width at half maximum (FWHM), the Fano factor $F=0.10$, and the resolution of the amplifier is $\Delta E_{\mathrm{amp}}=0.154 \mathrm{KeV} .{ }^{9} \epsilon$ here is the energy needed to generate an electron hole pair in germanium $(2.96 \mathrm{eV})$. A resolution of $26 \mathrm{eV}$ per channel was used. Keeping the deadtime of the detector below 5\%, the Lorentzian fraction of Voigt fits to the NBS640B silicon standard was determined to be $\leqslant 0.04$ independent of energy. ${ }^{4}$

The background was fitted using a third-order polynomial in the energy range 40-65 $\mathrm{keV}$ [for the (220), (311), and (222) reflections]. In the energy range $20-45 \mathrm{KeV}$ [for the (111) and (200) reflections], a third- or second-order polynomial added to a broad Gaussian were used. In this energy range, the intensity of the primary beam, the absorption of the diamonds, and the detector efficiency vary strongly.

\section{B. Analytical procedure}

Elastic uniaxial stresses acting on elastically anisotropic solids lead to lattice distortions. ${ }^{2}$ Hence, the lattice parameter (or volume) calculated from each reflection under such conditions is different. The uniaxial stress component $t\left(\equiv \sigma_{3}\right.$ $-\sigma_{1}$ where $\sigma_{3}$ and $\sigma_{1}$ are the stresses parallel and perdendicular to the load axis, respectively) superimposed on a hydrostatic stress can be calculated from the measured strains $\epsilon^{\text {total }}(h k l)$ with the relation derived using anisotropic elasticity theory: ${ }^{2}$

$$
\begin{aligned}
\epsilon^{\text {total }}(h k l)= & \epsilon^{p}-(1-\alpha) \frac{t}{3}\left(1-3 \sin ^{2} \theta\right) \frac{1}{2 \mu_{V}} \\
& -\alpha \frac{t}{3}\left(1-3 \sin ^{2} \theta\right)\left(S_{11}-S_{12}-3 S \Gamma\right),
\end{aligned}
$$

where $\epsilon^{\text {total }}(h k l)=\left[d(h k l)-d_{0}(h k l)\right] / d_{0}(h k l), \quad \epsilon^{p}$ is the hydrostatic strain, $V$ denotes the Voigt state (strain continuity), $\alpha$ is the fraction of the Reuss state (stress continuity) actually present in the sample, and $\mu$ is the shear modulus. $S$ is the elastic anisotropy factor $S=S_{11}-S_{12}-0.5 S_{44}$ and $\Gamma$ is the geometrical factor $\Gamma=\left(h^{2} k^{2}+k^{2} l^{2}+h^{2} l^{2}\right) /\left(h^{2}+k^{2}\right.$ $\left.+l^{2}\right)^{2}$. This equation is valid for a sample compressed elastically in an opposed anvil device with the incident beam along the compression axis. The product $\alpha t$ can be obtained from the slope $d \epsilon / d \Gamma$ when the elastic constants under pressure are known. Since the fraction $\alpha$ of the Reuss state cannot be determined with the geometry used here, ${ }^{10}$ the product $\alpha t$ is shown on all figures. In the present work, the elastic constants of $\mathrm{Cu}_{3} \mathrm{Au}$ under pressure were calculated from the experimentally determined values at ambient conditions ${ }^{11}$ with the pressure derivatives taken to be those determined for ordered $\mathrm{Cu}_{3} \mathrm{Au}$ up to $0.7 \mathrm{GPa}{ }^{12}$ The relative magnitude of the deviation from the hydrostatic value of $\left(d-d_{0}\right) / d_{0}$ for each lattice plane is determined by the factor $\left(S_{11}-S_{12}\right.$ $-3 S \Gamma$ ) (assuming a perfect Reuss state). For $\mathrm{Cu}_{3} \mathrm{Au}$, this factor decreases in the order $(200) \geqslant(311) \geqslant(220)$ $\geqslant(222,111)$ with values of $0.025,0.020,0.013$, and 0.008 $\mathrm{GPa}$ at ambient conditions and 0.040, 0.030, 0.013, and $0.003 \mathrm{GPa}$ at $30 \mathrm{GPa}$, respectively. Note that the shift due to uniaxial compressive stresses is positive in all cases, hence the observed volume in the presence of uniaxial compressive stresses should always be less than under hydrostatic conditions (assuming elastic response).

Above the yield point, disordered $\mathrm{Cu}_{3} \mathrm{Au}$ is known to deform by introduction of dislocations, stacking faults, and twin faults on $\{111\}$ planes. ${ }^{13-15}$ Introduction of dislocations leads to a volume expansion and line broadening. The volume expansion per unit length of a dislocation line is proportional to the ratios of the dilation energy (of the disloca- 
tion energy) to the bulk modulus and of the shear energy to the shear modulus. ${ }^{16}$ The dislocation density can be estimated with the approximation $\Delta V / V_{0} \approx 2 \rho b^{2}$, where $\rho$ is the dislocation density and $b$ is the Burger's vector ${ }^{16}$ (taken in the present work to be $1 / 2\langle 110\rangle)$. Outside their core radius, dislocations cause an elastic strain field. The strain broadening as observed with $\mathrm{x}$-ray diffraction depends on the dislocation density and the effective outer cutoff radius of dislocations. ${ }^{17}$ The dislocation density can be obtained with a Fourier analysis of measured linewidths; ${ }^{17-19}$ such analysis will not be attempted here because it requires high resolution probably not attainable with the energy-dispersive method.

The introduction of stacking faults on $\{111\}$ planes in fcc metals and superlattices leads to systematic shifts in peak positions. Transforming the equations given by Warren ${ }^{20}$ to the energy-dispersive case, the stacking fault probability $\alpha$ is related to the peak shift for the five reflections observed:

$$
\begin{aligned}
& \alpha=+\frac{\Delta E_{111}}{E_{111}} \frac{2 \pi}{\sqrt{3}} 42, \\
& \alpha=-\frac{\Delta E_{200}}{E_{200}} \frac{2 \pi}{\sqrt{3}} 22, \\
& \alpha=+\frac{\Delta E_{220}}{E_{220}} \frac{2 \pi}{\sqrt{3}} 42, \\
& \alpha=-\frac{\Delta E_{311}}{E_{311}} \frac{2 \pi}{\sqrt{3}} 112, \\
& \alpha=-\frac{\Delta E_{222}}{E_{222}} \frac{2 \pi}{\sqrt{3}} 82 .
\end{aligned}
$$

Equations (3) contain the strain as defined above (since $\Delta E / E=-\Delta d / d)$. However, $E_{h k l}$ in Eq. (3) is the peak position of an undeformed material (at a given pressure) whereas $d_{0}$ in Eq. (2) is the value at ambient conditions. The shift in $\Delta d / d$ for (200), (311), and (222) of $\mathrm{Cu}_{3} \mathrm{Au}$ due to the introduction of stacking faults is in the same direction as that due to elastic strains. For the other reflections, the shift due to plastic strains is in the direction opposite to that due to elastic strains. The shift is largest for (200).

The strains obtained from multiple orders of a reflection may be used to differentiate between elastic and plastic deformations. The elastic strain depends on the direction in the lattice only through the factor $S \Gamma$ [Eq. (2)]. The value of $\Gamma$ is the same for multiple orders of a reflection (and some other special directions in the lattice not observed here), and hence the strains must be the same. In the presence of stacking faults, different orders of a reflection are shifted by different amounts in opposite directions [Eq. (3)]. Therefore, different values of a strain or lattice parameter calculated from multiple orders of a reflection indicate the presence of plastic deformation. The introduction of stacking faults in $\mathrm{Cu}_{3} \mathrm{Au}$ leads to an overestimation of the uniaxial stress if calculated with Eq. (2). For a probability of stacking faults of 1 per 51 layers (for example), this would correspond to a compressive stress of $0.55 \mathrm{GPa}$ at $1 \mathrm{~atm}$; the $R$ value of the fit to $d \epsilon^{\text {total }} / d \Gamma$ in this case is only 0.848 .
Since the position resolution of the energy-dispersive method is $\Delta E / E \geqslant 5 \times 10^{-4}$, it is preferable to calculate the stacking fault probability from the peak separation between two reflections. ${ }^{20}$ By using multiple orders of a reflection, it is, in principle, possible to calculate the stacking fault probability free of contributions from elastic strains. Since the (222) reflection is rather weak (see above) and the shift for (222) is only $1 / 4$ that of (200) at a given stacking fault probability, the separation between (200) and (111) was used here:

$$
\alpha=-\Delta\left(E_{200}-E_{111}\right)\left(\frac{E_{200}}{2}+\frac{E_{111}}{4}\right)^{-1} \frac{2 \pi}{\sqrt{3}} 2,
$$

where $\Delta$ means the difference in peak separation after and before deformation. The peak separations should, in principle, be measured from the peak positions and not from the centroids of the peaks in order to avoid the influence of peak asymmetries due to twin faults. ${ }^{20}$ Since the difference in peak positions determined by these two methods was found to be at most $3 \times 10^{-4} \Delta E / E$ in the present study, the peak centroids were used. The values of the peak separation in the undeformed state under hydrostatic pressure were calculated using the equation of state parameters as stated above. The absolute values of the stacking fault probability should therefore be regarded as estimates only.

Stacking faults and twin faults contribute to peak broadening like a size effect. ${ }^{20}$ This contribution is $a c 1.5 /(\alpha$ $+\beta)=L^{\prime}$ where $a$ is the lattice parameter, $\beta$ is the twin fault probability, $c$ is a constant which depends on $h k l$, and $L^{\prime}$ is the size. ${ }^{20}$ These relations were derived from a Fourier analysis of peak broadening without assumption about peak shapes. The effective crystallite size (which includes the contribution $L^{\prime}$ from faulting) is given by the inverse of the offset in a plot of the Fourier coefficients versus multiple orders of a reflection. Twin faults lead in addition to a peak asymmetry. ${ }^{20}$ Such asymmetries, if restricted to the tails of a reflection, may be masked by effects from the solid-state detector and its electronics. ${ }^{4}$

It was observed in the previous study of a $\mathrm{Cu}_{3} \mathrm{Au}$ foil in a diamond-anvil cell with $\mathrm{NaCl}$ as a pressure medium ${ }^{5}$ and in the present work that the peak profiles of $\mathrm{Cu}_{3} \mathrm{Au}$ had a Lorentzian component in addition to a Gaussian one even at ambient pressure. Since the Lorentzian fraction was found to increase under pressure, ${ }^{5}$ Voigt fits were employed for all reflections observed in the previous and the present study. The variation with pressure of the line profile is illustrated here with the Lorentzian $\left(\beta_{C}\right)$ and Gaussian $\left(\beta_{G}\right)$ integral widths and the Lorentzian fraction $\left(\beta_{C} / \beta\right)$ (all corrected for the instrumental resolution) and the uncorrected FWHM. ${ }^{4}$ The integral width $\beta$ is given by area divided by intensity and hence depends sensitively on the background. The Lorentzian fraction and Lorentzian and Gaussian integral widths can be calculated from $\beta$ and the FWHM with an approximation accurate to $1 \%::^{21}$

$$
\beta_{C}=\beta\left(2.0207-0.4803 \phi-1.7756 \phi^{2}\right),
$$

$$
\beta_{G}=\beta\left(0.6442+1.4187 \sqrt{\phi-2 / \pi}-2.2043 \phi+1.8706 \phi^{2}\right),
$$


where $\phi$ is the FWHM divided by the integral width $\beta$. The FWHM is the parameter least affected by how the background is fitted. (It should be noted that the parameter $\phi$ varies between 0.9395 for a pure Gaussian and 0.6366 for a pure Lorentzian. ${ }^{21}$ Gaussian and Lorentzian fractions thus do not add to 1.0.)

As discussed above, line profiles, in principle, contain information about both size and strain. ${ }^{22,23}$ When multiple orders of a reflection are available, the strain and size contributions can be separated by the Williamson-Hall ${ }^{24}$ or the Warren-Averbach ${ }^{25}$ method (the latter of which uses a Fourier analysis of line profiles; it does not make any assumption about profile shapes). When this is not the case, strain and size are commonly taken to cause Gaussian and Lorentzian broadening, respectively, of individual reflections. ${ }^{21,23,26}$ With these assumptions, the effective crystallite size ( $\left.L_{\text {eff }}\right)$ and the strain $(\epsilon)$ can be determined from the measured integral widths: ${ }^{27}$

$$
\begin{gathered}
\beta_{C}=\frac{6.199}{L_{\text {eff }} \sin \theta_{0}}, \\
\beta_{G}=2 \epsilon E,
\end{gathered}
$$

where $\theta_{0}$ is half the diffraction angle. The effective crystallite size is a volume average of the effective size perpendicular to the reflecting planes. While the single line method of profile analysis to be used here and the Fourier method yield different estimates of size and strain, ${ }^{23,28}$ both the effective particle size calculated with the above equation and an estimate of the true size calculated from this equation after correction for the crystallite size effect of stacking faults will be presented for comparison.

\section{RESULTS}

\section{A. Compression in 4:1 methanol-ethanol}

The compression behavior is illustrated with the scaled volumes calculated from the (111) and (200) lattice spacings (Fig. 1). This representation was chosen because the scaled volumes for all lattice spacings should coincide for hydrostatic compression and this is what is usually plotted in equation-of-state studies. While a deviation from an equation of state of an average volume calculated from all observed reflections is in itself an indication of uniaxial stresses, the deviations of the scaled volume calculated from individual lattice spacings allow for a quantitative determination of elastic and plastic strains. For the five reflections observed, the scaled volumes of (111) and (200) should represent the extreme values in the case of both elastic and plastic strains [Sec. II A, Eqs. (2) and (3)]. The compressional behavior for all lattice spacings observed is very similar as is easily seen from Table I; differences are to be discussed below. The scaled average volumes for all experiments will be plotted for comparison in part II.

A smooth compression to about $7 \mathrm{GPa}$ is followed by hardening to just under $12 \mathrm{GPa}$ (Fig. 1). The scaled average volume remains unchanged between 12 and $13 \mathrm{GPa}$ (compare Table I). At $13 \mathrm{GPa}$, there is a kink followed by smooth compression up to the highest pressure reached. The scaled volume calculated from the (200) lattice spacing deviates

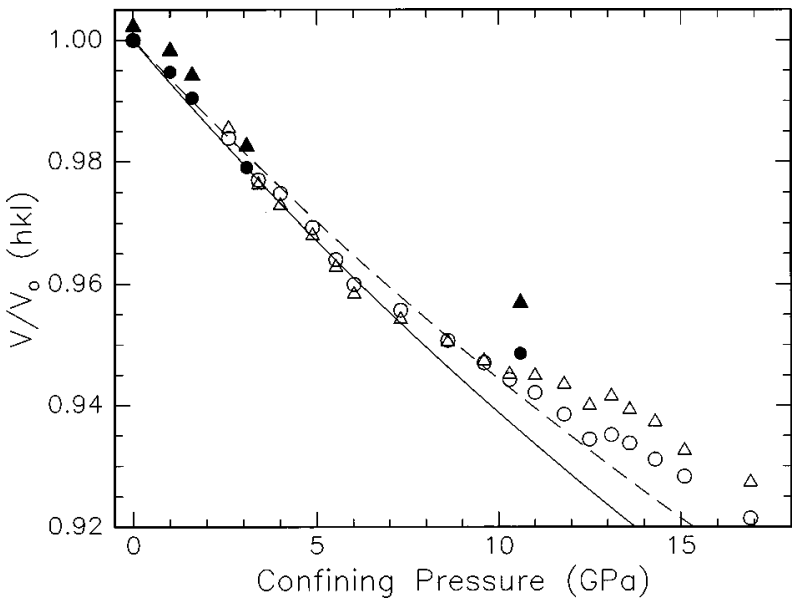

FIG. 1. The scaled volumes calculated from the (111) (circles) and (200) (triangles) lattice spacings of $\mathrm{Cu}_{3} \mathrm{Au}$ in 4:1 methanolethanol versus pressure. Open symbols are for increasing confining pressure and solid symbols are for pressure release. The solid line represents the first-order Birch-Murnaghan equation of state fitted to the data to $7 \mathrm{GPa}$. The bulk modulus thus determined agrees very well with the isothermal bulk modulus calculated from the ultrasonically determined data at ambient conditions (Ref. 11). The dashed line represents a second-order Birch-Murnaghan equation of state using the pressure derivative of the bulk modulus determined ultrasonically on ordered $\mathrm{Cu}_{3} \mathrm{Au}$ up to $0.7 \mathrm{GPa}\left(B_{0}^{\prime}=5.96\right)$ (Ref. 12).

towards values higher than those calculated from the other reflections starting at around $7 \mathrm{GPa}$. This deviation becomes very strong at around $10 \mathrm{GPa}$ and remains constant above 13 $\mathrm{GPa}$ (Fig. 1). A fit to the scaled average volume below $7 \mathrm{GPa}$ with a first-order Birch-Murnaghan ${ }^{29}$ equation of state yields a bulk modulus of $139.6 \pm 2 \mathrm{GPa}$. The agreement with the isothermal bulk modulus calculated from the elastic constants determined at ambient conditions ${ }^{11}\left(B_{0}=140.6 \mathrm{GPa}\right)$ is excellent. On pressure release, the hysteresis of the elastic volume strain closes somewhere between 11 and $3 \mathrm{GPa}$ [the hysteresis loop was not followed in detail because it was expected to close at much lower pressure, as observed for $\mathrm{Cu}_{3} \mathrm{Au}$ in $\mathrm{NaCl}$ (Ref. 5)]. Note that the difference in volumes calculated from the (200) and the other reflections is retained from high pressures even at ambient pressure, and that the lattice spacing for (200) is larger after the pressure cycle than before (see Table I).

The stresses (or rather the product $\alpha t$ ) calculated from the observed line shifts of the reflections remain essentially constant to $7 \mathrm{GPa}$ (Fig. 2). There is a strong increase in the compressive stress between 7 and around $12 \mathrm{GPa}$ at which pressure the stresses saturate with values of around 0.25 $\mathrm{GPa}$. On pressure release, the stresses do not relax.

The behavior of the line profile is illustrated here with the (220) reflection only (Fig. 3). The behavior of the FWHM of the observed reflections is similar (except for the absolute changes). Changes in the FWHM of the (111) reflection were too small to be analyzed in terms of Gaussian and Lorentzian contributions. The calculated Gaussian and Lorentzian contributions to the (311) and (222) reflections are not considered reliable because these reflections overlap with the tungsten fluorescence (from the collimators and slits) and their intensities are rather weak. The FWHM of the observed re- 
TABLE I. $d$ spacings as a function of pressure for compression of the $\mathrm{Cu}_{3} \mathrm{Au}$ foil in 4:1 methanol-ethanol.

\begin{tabular}{|c|c|c|c|c|c|}
\hline \multirow{2}{*}{$\begin{array}{c}\text { Pressure }(\mathrm{GPa}) \\
h k l\end{array}$} & \multicolumn{5}{|c|}{$d$ spacings $(\AA)$} \\
\hline & 111 & 200 & 220 & 311 & 222 \\
\hline 0.0001 & 2.170 & 1.879 & 1.329 & 1.134 & 1.085 \\
\hline 2.6 & 2.158 & 1.870 & 1.322 & 1.127 & 1.079 \\
\hline 3.4 & 2.153 & 1.864 & 1.319 & 1.125 & 1.077 \\
\hline 4.0 & 2.152 & 1.862 & 1.317 & 1.124 & 1.076 \\
\hline 4.9 & 2.148 & 1.859 & 1.315 & 1.122 & 1.074 \\
\hline 5.5 & 2.144 & 1.855 & 1.313 & 1.119 & 1.072 \\
\hline 6.0 & 2.141 & 1.853 & 1.311 & 1.118 & 1.071 \\
\hline 7.3 & 2.138 & 1.850 & 1.309 & 1.116 & 1.069 \\
\hline 8.6 & 2.134 & 1.847 & 1.306 & 1.114 & 1.067 \\
\hline 9.6 & 2.131 & 1.845 & 1.305 & 1.113 & 1.066 \\
\hline 10.3 & 2.129 & 1.844 & 1.304 & 1.112 & 1.065 \\
\hline 11.0 & 2.127 & 1.844 & 1.303 & 1.112 & 1.064 \\
\hline 11.8 & 2.125 & 1.843 & 1.302 & 1.110 & 1.063 \\
\hline 12.5 & 2.122 & 1.841 & 1.300 & 1.109 & 1.061 \\
\hline 13.1 & 2.122 & 1.842 & 1.300 & 1.109 & 1.061 \\
\hline 13.6 & 2.121 & 1.840 & 1.299 & 1.109 & 1.061 \\
\hline 14.3 & 2.119 & 1.839 & 1.298 & 1.108 & 1.061 \\
\hline 15.1 & 2.117 & 1.836 & 1.297 & 1.106 & 1.059 \\
\hline 16.9 & 2.112 & 1.832 & 1.293 & 1.104 & 1.056 \\
\hline 10.6 & 2.132 & 1.852 & 1.306 & 1.115 & 1.066 \\
\hline 3.1 & 2.155 & 1.868 & 1.320 & 1.126 & 1.078 \\
\hline 1.6 & 2.163 & 1.875 & 1.325 & 1.130 & 1.082 \\
\hline 1.0 & 2.166 & 1.878 & 1.326 & 1.132 & 1.083 \\
\hline 0.0001 & 2.170 & 1.880 & 1.329 & 1.133 & 1.085 \\
\hline
\end{tabular}

flections increase from ambient conditions to a maximum in the range $3-5 \mathrm{GPa}$ (for 111,200 , and 222) or $7 \mathrm{GPa}(220$, top of Fig. 3, and 311). They relax up to pressures of 10-12 $\mathrm{GPa}$ in which pressure range they increase again to the level of the maximum at lower pressures. The Lorentzian fraction for the (220) reflection at ambient conditions appears to be too high possibly masking an increase in $\beta_{C} / \beta$ at low pressure. It appears to remain constant between 2.6 and just below $10 \mathrm{GPa}$ (Fig. 3, middle). It increases from about 0.4 to

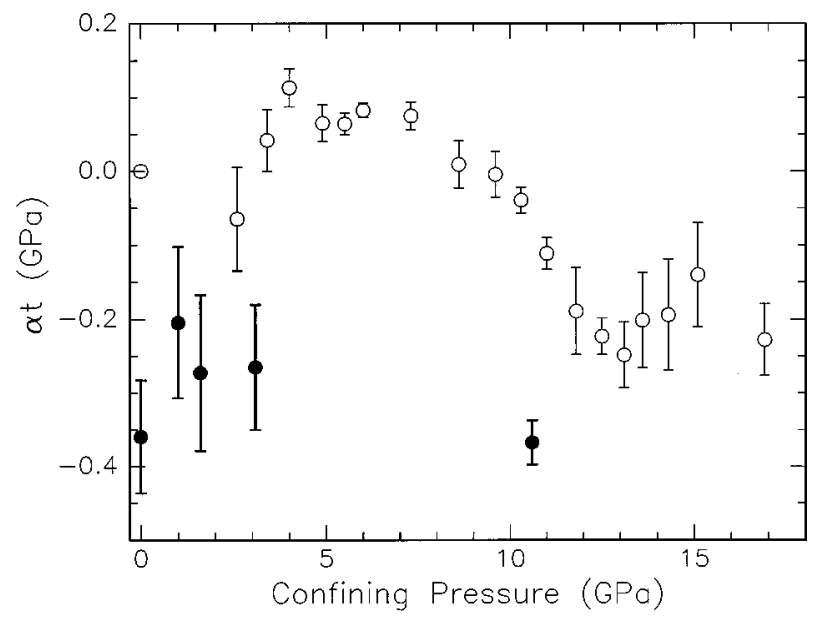

FIG. 2. The product $\alpha t$ for $\mathrm{Cu}_{3} \mathrm{Au}$ in 4:1 methanol-ethanol with increasing confining pressure (open circles) and pressure release (solid circles). The error bars are from the fit to the strain versus direction in the lattice (Ref. 2).
0.5 between 10 and $12 \mathrm{GPa}$ and remains constant thereafter. There is no relaxation from this value on pressure release. The data for the (200) reflection scatter more strongly and hence the trend is not clear apart from an absolute increase

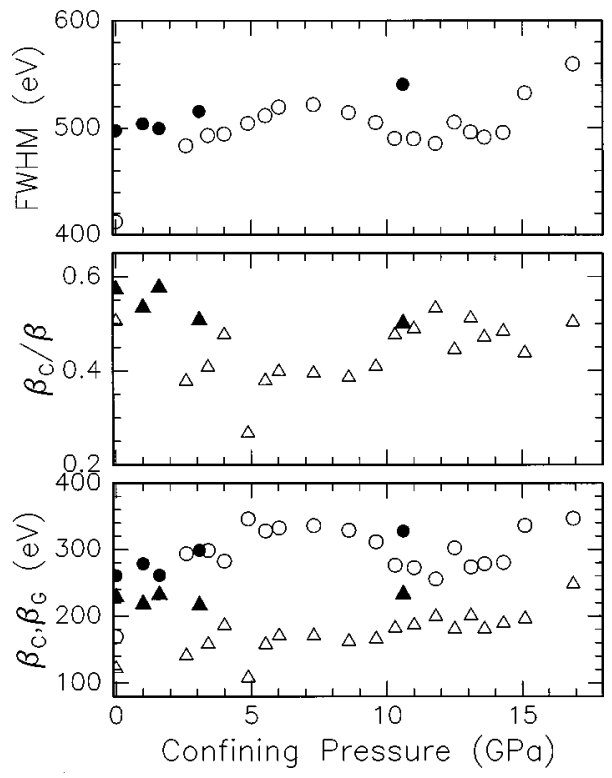

FIG. 3. Voigt fit parameters to the (220) diffraction peak of $\mathrm{Cu}_{3} \mathrm{Au}$. FWHM (top), Lorentzian fraction (middle), and Gaussian $\left(\beta_{G}\right.$, circles) and Lorentzian $\left(\beta_{C}\right.$, triangles) integral widths (bottom). Open symbols are for pressure increase and solid symbols for pressure release. 


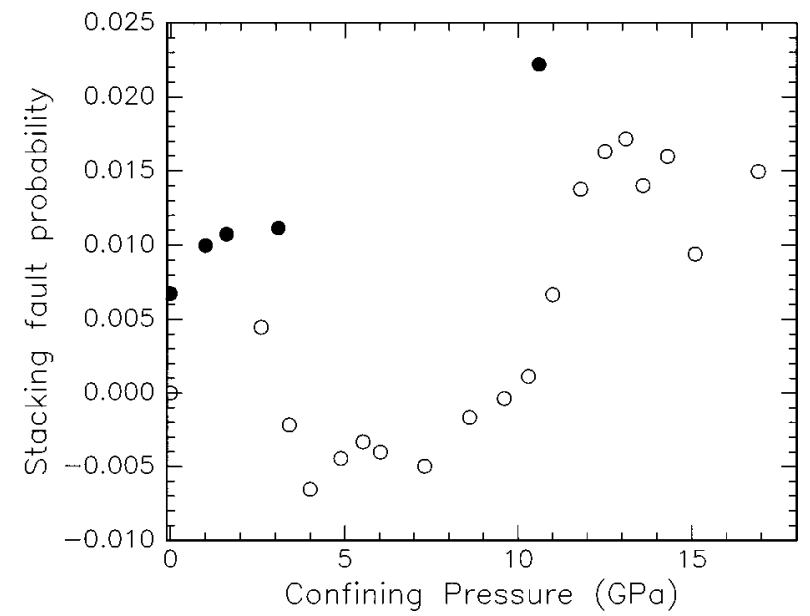

FIG. 4. The stacking fault probability calculated from the relative peak separation of the (111) and (200) reflections as a function of pressure. Open circles are for pressure increase, and solid circles for pressure release. The minimum probability that can be detected with the energy-dispersive method is estimated to be around 0.005 .

due to the pressure cycle, and no relaxation on pressure release. The Lorentzian integral widths for (200) and (220) (Fig. 3, bottom) follow the trend for the Lorentzian fraction of (220). The Gaussian integral widths for these two reflections follow the trends of the respective FWHM [see bottom of Fig. 3 for the trend of the (220) reflection]. Some relaxation was found on pressure release only for the Gaussian integral width of the (220) reflection.

The stacking fault probability as a function of pressure was calculated from the separation of the (111) and (200) reflections assuming that the strains are entirely plastic, as explained above. A rise in the probability of stacking faults at $7 \mathrm{GPa}$ and its saturation at $12 \mathrm{GPa}$ can be seen in Fig. 4. On pressure release, the stacking fault probability seems to decrease.

\section{B. Compression in paraffin oil}

The compression curve (Fig. 5) shows the same features already seen with $\mathrm{NaCl}$ (Ref. 5) and methanol-ethanol as the pressure transmitting media. (For reasons of clarity, the data are shown only up to a pressure of $16 \mathrm{GPa}$. The complete data are given in Tables II and III. An offset in the data at 20 $\mathrm{GPa}$ and a slightly different slope of the compression curve above this pressure is presumably due to relaxation effects [compare also the stress curve, Fig. 6(b)] and may also include effects resulting from recalibration and realignment; see Fig. 10, part II.) After an initially high compressibility, there is a pressure interval $(5-7 \mathrm{GPa})$ over which the scaled average volume remains constant (no elastic compression). A discontinuity in the compressibility at $7 \mathrm{GPa}$ is followed by isotropic compression with a compressibility similar to the initial one. Note that the volume calculated from the (200) reflection starts to deviate towards values higher than those calculated from the other reflections at around 5-6 $\mathrm{GPa}$. The magnitude of the deviation generated in this pressure range remains unchanged above about $7 \mathrm{GPa}$. The agreement between the two experiments carried out (shown by open and solid symbols, respectively) demonstrates that the results are reproducible.

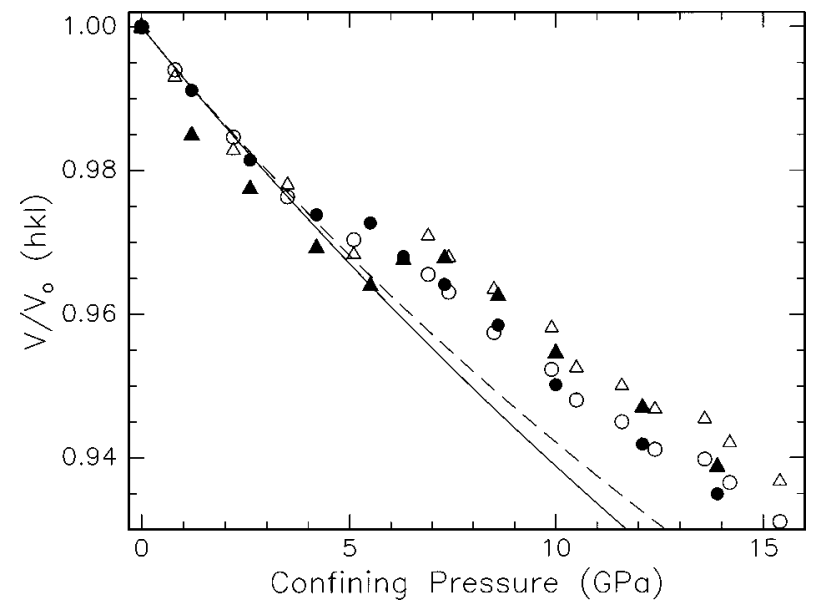

FIG. 5. The scaled volume calculated from the (111) and (200) reflections of $\mathrm{Cu}_{3} \mathrm{Au}$ (circles and triangles, respectively). Open and solid symbols represent two different experiments. The solid line is a first-order Birch-Murnaghan equation of state fitted to the data of $\mathrm{Cu}_{3} \mathrm{Au}$ in 4:1 methanol-ethanol $\left(B_{0}=139.6 \mathrm{GPa}\right)$. The dashed line is a second-order Birch-Murnaghan equation of state using the isothermal bulk modulus $\left(B_{0}=140.6 \mathrm{GPa}\right)$ calculated from the elastic constants of disordered $\mathrm{Cu}_{3} \mathrm{Au}$ determined at ambient conditions (Ref. 11) and the pressure derivative of the bulk modulus $B_{0}^{\prime}$ $=5.96$ determined ultrasonically for ordered $\mathrm{Cu}_{3} \mathrm{Au}$ up to $0.7 \mathrm{GPa}$ (Ref. 12).

The compressive stresses calculated from the relative line shifts in $\mathrm{Cu}_{3} \mathrm{Au}$ in both experiments saturate at a value of $0.25 \mathrm{GPa}$ at around $7 \mathrm{GPa}$ [near the kink in the compression curve; Figs. 6(a,b)]. Positive values of the stress were observed in both experiments to $5.5 \mathrm{GPa}$. Their magnitude in the second experiment is zero within experimental error. The value at 5.1 GPa during the second experiment can be explained from a strong asymmetry in the (200) reflection. The rather large positive values of the stress to $5.5 \mathrm{GPa}$ during the first experiment can be explained with the high initial value of the (200) compared with the other lattice spacings (Table I). The high initial value of (200) results in a strain (a compressibility) which is apparently larger for (200) than for the other lattice spacings. Since $\Gamma(200)$ and $\Gamma(111)$ are the extreme values for the observed lattice spacings and since only five reflections were observed, the strain values for (111) and (200) may determine the sign and magnitude of $d \epsilon / d \Gamma[\mathrm{Eq} .(2)]$. In such a case, if the measured strains are larger for (200) than for (111) and with the appropriate sign of $S$, a positive value of the stress can result.

The offset in the stress and volume data at $20 \mathrm{GPa}$ may be due to relaxation (the stresses before and after the break are 0.25 and $0.1 \mathrm{GPa}$, respectively), with unknown contributions from recalibration/realignment of the diffraction setup after the storage ring crashed. On pressure release, there is only a minor relaxation of stresses to residual compressive stresses of around $0.15 \mathrm{GPa}$.

A calculation of the stacking fault probability was not attempted because of the limited data of both runs and the experimental difficulties of the second run. In addition, there were strong asymmetries in the (200) reflection at 4.2, 5.1, and 6.3 GPa (first run) and 5.1 GPa (second run) which make it difficult to determine peak positions accurately. 
TABLE II. $d$ spacings as a function of pressure for compression of $\mathrm{Cu}_{3} \mathrm{Au}$ foil in paraffin oil (first run).

\begin{tabular}{|c|c|c|c|c|c|}
\hline \multirow{2}{*}{$\begin{array}{c}\text { Pressure (GPa) } \\
h k l\end{array}$} & \multicolumn{5}{|c|}{$d$ spacings $(\AA)$} \\
\hline & 111 & 200 & 220 & 311 & 222 \\
\hline 0.0001 & 2.170 & 1.882 & 1.329 & 1.133 & 1.085 \\
\hline 1.2 & 2.163 & 1.872 & 1.325 & 1.130 & 1.082 \\
\hline 2.6 & 2.156 & 1.867 & 1.320 & 1.126 & 1.078 \\
\hline 4.2 & 2.151 & 1.862 & 1.317 & 1.124 & 1.075 \\
\hline 5.5 & 2.150 & 1.859 & 1.316 & 1.123 & 1.074 \\
\hline 6.3 & 2.146 & 1.861 & 1.316 & 1.123 & 1.074 \\
\hline 7.3 & 2.144 & 1.861 & 1.313 & 1.121 & 1.073 \\
\hline 8.6 & 2.139 & 1.858 & 1.311 & 1.119 & 1.071 \\
\hline 10.0 & 2.133 & 1.853 & 1.307 & 1.116 & 1.068 \\
\hline 12.1 & 2.127 & 1.848 & 1.303 & 1.113 & 1.065 \\
\hline 13.9 & 2.122 & 1.842 & 1.300 & 1.110 & 1.062 \\
\hline 0.0001 & 2.169 & 1.882 & 1.329 & 1.134 & 1.085 \\
\hline
\end{tabular}

The fit parameters of the Voigt functions are illustrated in Figs. 7,8 $(\mathrm{a}-\mathrm{d})$. For the first run, the parameters for the (220) reflection only are shown as a representative example. The trend in the (111) reflection is not clear since the broadening is quite small [this was also observed in the case of $\mathrm{NaCl}$ (Ref. 5) and 4:1 methanol-ethanol pressure transmitting media; but compare (111) for the second run, Fig. 8(a)]. The (200) reflection was markedly asymmetric in the pressure range 4.2-6.3 GPa and the limited data set is not shown. The
(222) reflection is not shown for either run because it is too weak and overlaps with the tungsten fluorescence from the collimators and slits.

The trends in the Voigt fit parameters from both runs agree (saturation in any parameter was, however, not reached during the first run). There is a small increase to $2 \mathrm{GPa}$ in the FWHM [top of Figs. 7,8 (a-d)] and the Lorentzian and Gaussian integral widths [bottom of Figs. 7,8(a-d)]. The initial values of the Lorentzian integral widths appear to be too

TABLE III. $d$ spacings as a function of pressure for compression of $\mathrm{Cu}_{3} \mathrm{Au}$ foil in paraffin oil (second run).

\begin{tabular}{|c|c|c|c|c|c|}
\hline \multirow{2}{*}{$\begin{array}{c}\text { Pressure (GPa) } \\
h k l\end{array}$} & \multicolumn{5}{|c|}{$d$ spacings $(\AA)$} \\
\hline & 111 & 200 & 220 & 311 & 222 \\
\hline 0.0001 & 2.171 & 1.881 & 1.330 & 1.134 & 1.086 \\
\hline 0.8 & 2.166 & 1.876 & 1.328 & 1.132 & 1.083 \\
\hline 2.2 & 2.159 & 1.870 & 1.322 & 1.128 & 1.080 \\
\hline 3.5 & 2.153 & 1.867 & 1.320 & 1.125 & 1.077 \\
\hline 5.1 & 2.149 & 1.861 & 1.317 & 1.123 & 1.075 \\
\hline 6.9 & 2.145 & 1.862 & 1.315 & 1.122 & 1.074 \\
\hline 7.4 & 2.144 & 1.860 & 1.314 & 1.121 & 1.072 \\
\hline 8.5 & 2.139 & 1.858 & 1.311 & 1.120 & 1.071 \\
\hline 9.9 & 2.136 & 1.854 & 1.309 & 1.118 & 1.069 \\
\hline 10.5 & 2.132 & 1.851 & 1.307 & 1.116 & 1.067 \\
\hline 11.6 & 2.130 & 1.849 & 1.306 & 1.115 & 1.066 \\
\hline 12.4 & 2.127 & 1.847 & 1.304 & 1.114 & 1.064 \\
\hline 13.6 & 2.126 & 1.846 & 1.303 & 1.113 & 1.064 \\
\hline 14.2 & 2.124 & 1.844 & 1.301 & 1.111 & 1.062 \\
\hline 15.4 & 2.120 & 1.840 & 1.299 & 1.109 & 1.060 \\
\hline 16.7 & 2.115 & 1.836 & 1.296 & 1.107 & 1.058 \\
\hline 18.2 & 2.110 & 1.830 & 1.293 & 1.104 & 1.056 \\
\hline 20.0 & 2.103 & 1.823 & 1.287 & 1.099 & 1.053 \\
\hline 19.9 & 2.101 & 1.822 & 1.286 & 1.098 & 1.051 \\
\hline 22.4 & 2.093 & 1.816 & 1.281 & 1.094 & 1.048 \\
\hline 24.5 & 2.088 & 1.812 & 1.279 & 1.093 & 1.045 \\
\hline 26.7 & 2.083 & 1.808 & 1.276 & 1.090 & 1.042 \\
\hline 28.7 & 2.078 & 1.804 & 1.273 & 1.087 & 1.040 \\
\hline 29.9 & 2.074 & 1.801 & 1.271 & 1.085 & 1.037 \\
\hline 0.0001 & 2.170 & 1.881 & 1.329 & 1.134 & 1.085 \\
\hline
\end{tabular}




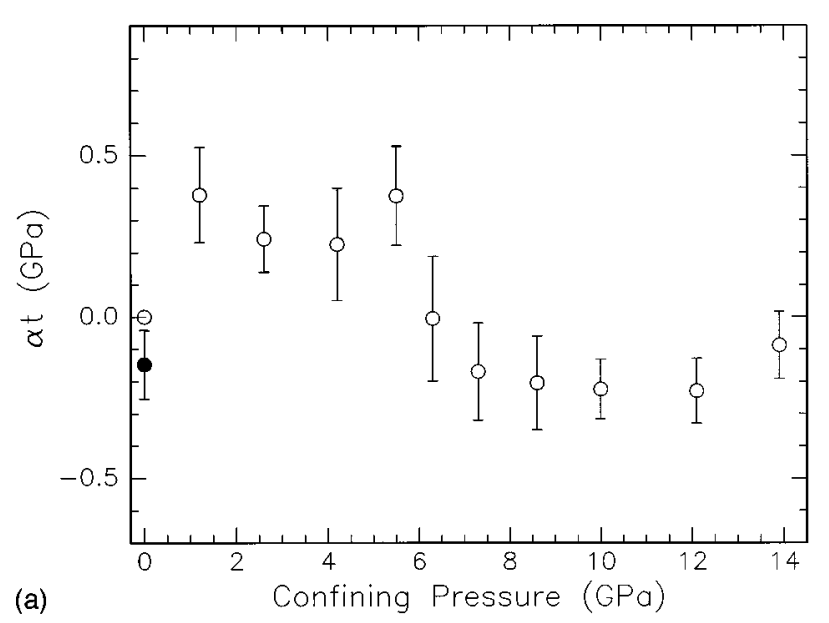

(a)

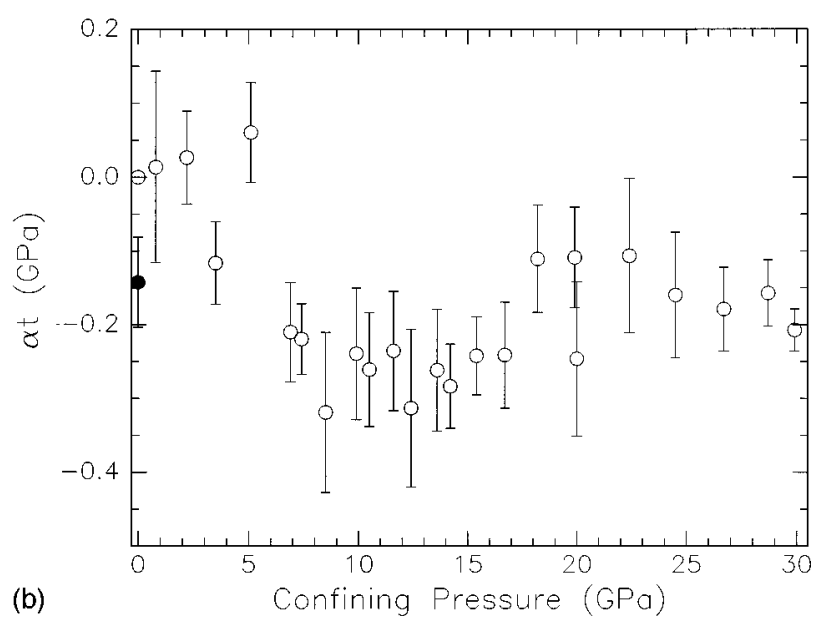

FIG. 6. The product $\alpha t$ (fraction of Reuss state in the sample times the uniaxial stress) for runs 1 (a) and 2 (b). Solid symbols are for pressure release. Note the residual stress of $0.15 \mathrm{GPa}$.

high relative to the Gaussian ones except for (200) [middle part of Figs. 7,8(a-d)]. There is a fair amount of scatter in these data below $5 \mathrm{GPa}$. The FWHM and Lorentzian and Gaussian integral widths do not change between 2 and 5 $\mathrm{GPa}$. At this pressure, there is a strong increase up to 12 or $18 \mathrm{GPa}$. The FWHM of the Voigt functions saturate at 12 GPa [Figs. 8(a,b)] or 18-20 GPa [Figs. 8(c,d)]. Note that the FWHM of all reflections show some relaxation around 12 $\mathrm{GPa}$ (at the pressure at which this run was interrupted due to the power failure). The FWHM of the (220) and (311) reflections continue to increase above this pressure. The relaxation for (111) and (200) is also seen in $\beta_{G}$ [bottom of Figs. $8(\mathrm{a})$ and $8(\mathrm{~b})]$. The Lorentzian fractions saturate in the range $10 \mathrm{GPa}$ [for (220) of the first run and (311) of the second run] to $18 \mathrm{GPa} . \beta_{C} / \beta$ for all reflections increases from values around $0.45-0.5$ below $5 \mathrm{GPa}$ to $0.6-0.7$. [The behavior for the uncorrected Lorentzian fraction is more regular than for the corrected one. In particular, the rapid increase at 5 $\mathrm{GPa}$ and the saturation at around $18 \mathrm{GPa}$ is observed to clearly take place also for (111) and (220), as for (200).] Gaussian and Lorentzian integral widths (open circles and triangles, respectively) are similar to each other at ambient conditions [except for a difference of $\approx 50 \mathrm{eV}$ in (220) of the first run and (111) and (200) of the second run]. Lorentzian and Gaussian integral widths at ambient conditions are around 150 and $200 \mathrm{eV}$, respectively, for all reflections ex-

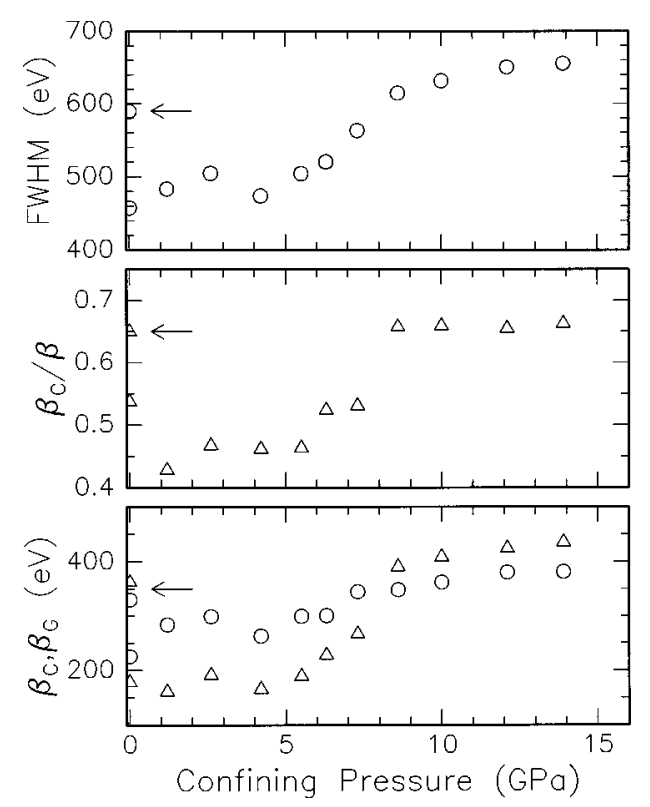

FIG. 7. Voigt fit parameters to the (220) reflection of run 1. At the top are the FWHM of the Voigt function, the middle figure is for the Lorentzian fraction $\beta_{C} / \beta$, and at the bottom are the Gaussian (circles) and Lorentzian (triangles) integral widths. Arrows indicate data taken on pressure release. The anomalously large starting value of $\beta_{C} / \beta$ is due to a rather high initial Lorentzian width.

cept for (111) of the second run where $\beta_{C} \approx 70 \mathrm{eV}$ and $\beta_{G}$ $\approx 20 \mathrm{eV}$ [bottom of Figs. 7,8(a-d)]. The increase in Gaussian and Lorentzian integral widths is similar in magnitude; they saturate at around $18 \mathrm{GPa}$ except for the Gaussian integral widths of the (111) and (200) reflections of the second run which appear to be affected by relaxation. On pressure release from $30 \mathrm{GPa}$, there is virtually no relaxation in any fit parameters, showing that the deformation (as recorded by the FWHM) is almost entirely plastic.

Peak asymmetries which may indicate the presence of twin faults ${ }^{20}$ were not detected except between 4.2 and 6.3 GPa during the first run and at 5.1 GPa during the second run. No quantitative evaluation was attempted because of the strongly varying background underneath the peaks.

Since the effective crystallite size and strain data simply repeat the trends of the integral widths from which they are calculated using Eqs. (7) and (8), no graphs are shown. Some reference values (for ambient conditions before and after the run, and for $29.9 \mathrm{GPa}$ ) are presented in Table IV (for the second run only). While the effective size is largest and strain lowest for the $\langle 111\rangle$ direction, the trends are similar for all reflections. The effective size is reduced roughly in half (for 111) or by $1 / 3$ by the application of $30 \mathrm{GPa}$ pressure, and the strain is increased by a factor between 2 (for 200) and 3 (for 220 and 311). It is increased by a factor of 10 for (111).

\section{DISCUSSION}

The compression curves (Figs. 1,5) show the features expected for initially undeformed elastically anisotropic polycrystals under increasing nonhydrostatic stress. ${ }^{5}$ The average volume data for $\mathrm{Cu}_{3} \mathrm{Au}$ in 4:1 methanol-ethanol to $7 \mathrm{GPa}$ and in paraffin oil to $4 \mathrm{GPa}$ can be fitted well with a first-order 

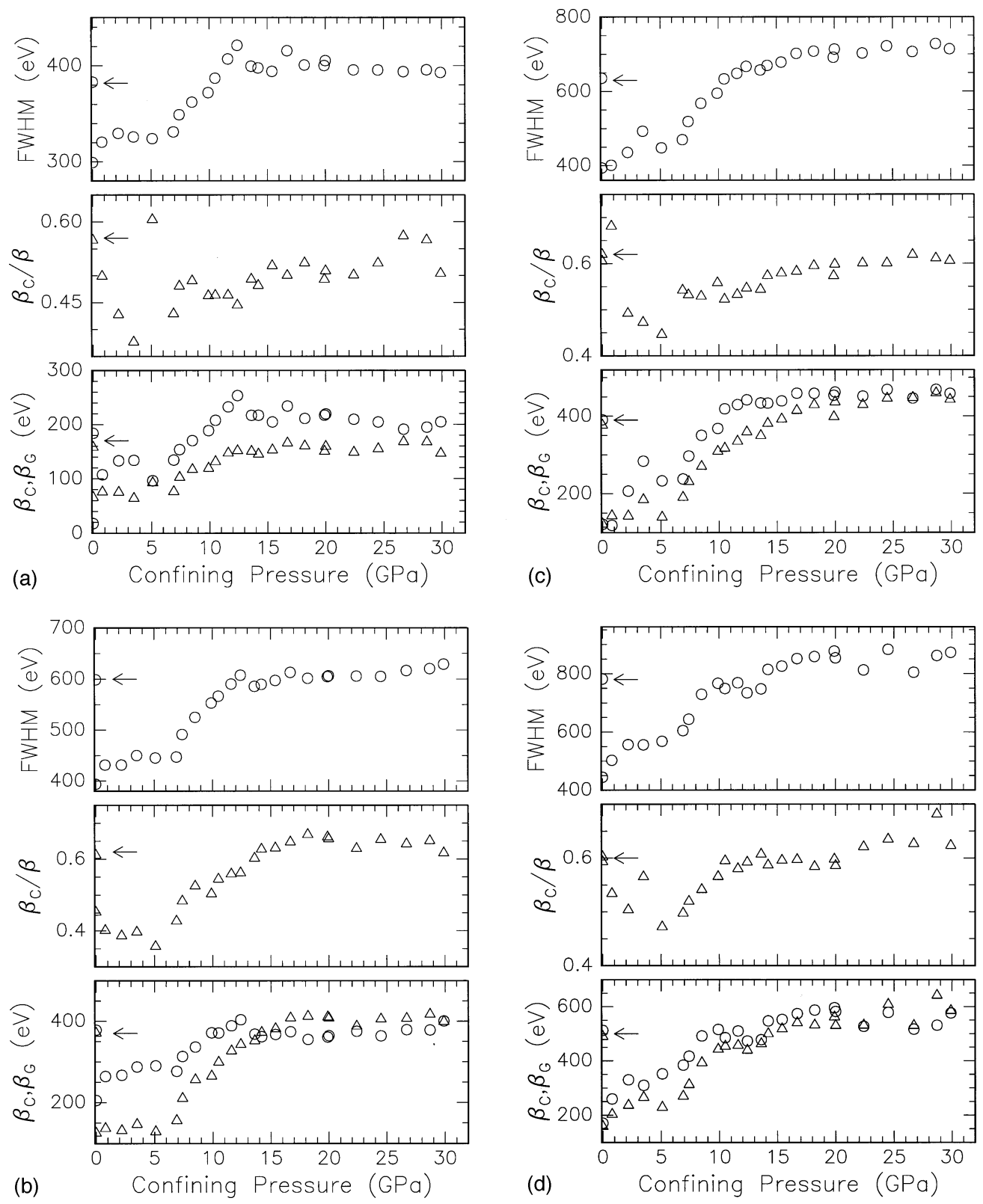

FIG. 8. Voigt fit parameters to the (111) (a), (200) (b), 220 (c) and 311 (d) reflections of run 2. Symbols as for Fig. 7. The initial value of $\beta_{C} / \beta$ for (111) is off scale as can be seen from the relative values of $\beta_{C}$ and $\beta_{G}$ [bottom of (a)]. The initial values of $\beta_{C} / \beta$ for (111), (220), and (311) are anomalously high.

Birch-Murnaghan equation of state using the ultrasonically determined bulk modulus. ${ }^{11}$ The data lie below those calculated with a second-order Birch-Murnaghan equation of state using this value and the pressure derivative determined for ordered $\mathrm{Cu}_{3} \mathrm{Au}\left(B_{0}^{\prime}=5.96\right) .{ }^{12}$ While the increase in the
FWHM immediately on first compression (up to 2-3 GPa in paraffin oil, Figs. 7,8; up to $7 \mathrm{GPa}$ in methanol-ethanol, Fig. 3 ) indicates the presence of strains, the calculated stresses are rather small ( $\leqslant 0.1 \mathrm{GPa}$ to 2 and $7 \mathrm{GPa}$ in the case of paraffin oil and methanol-ethanol, respectively). No evidence for

TABLE IV. Effective size and strain calculated from the Lorentzian and Gaussian integral widths (Refs. 12-15), respectively, for ambient conditions before and after the run, and for $29.9 \mathrm{GPa}$ of the second run with paraffin oil.

\begin{tabular}{|c|c|c|c|c|c|c|c|c|}
\hline \multirow{2}{*}{$\begin{array}{l}\text { Pressure (GPa) } \\
h k l\end{array}$} & \multicolumn{4}{|c|}{ Effective size $(\AA)$} & \multicolumn{4}{|c|}{ Strain $\left(10^{-4}\right)$} \\
\hline & 111 & 200 & 220 & 311 & 111 & 200 & 220 & 311 \\
\hline 0.0001 & 998 & 522 & 536 & 412 & 3 & 29 & 12 & 15 \\
\hline 29.9 & 447 & 164 & 148 & 112 & 32 & 55 & 45 & 48 \\
\hline 0.0001 & 414 & 176 & 174 & 134 & 31 & 54 & 40 & 44 \\
\hline
\end{tabular}


stacking faults on $\{111\}$ planes was detected to $7 \mathrm{GPa}$ in $4: 1$ methanol-ethanol (Fig. 4), but the resolution of the energydispersive method requires more than roughly 1 stacking fault per 200 layers for detection. From the good agreement in the lattice parameters calculated from the individual reflections in the experiment and the excellent agreement in the average volume among the data sets, it is concluded that the initial compression is hydrostatic up to $7 \mathrm{GPa}$ in $4: 1$ methanol-ethanol within the resolution of the experimental method. The upper limit of hydrostaticity of paraffin oil was not clearly observed because the deviation from the equation of state at $5 \mathrm{GPa}$ corresponds to the one seen in 4:1 methanol-ethanol at $12 \mathrm{GPa}$. The hydrostatic limit of paraffin oil with respect to polycrystalline $\mathrm{Cu}_{3} \mathrm{Au}$ must therefore lie well below $5 \mathrm{GPa}$. The deviation from the equation of state in 4:1 methanol-ethanol above $7 \mathrm{GPa}$ is seen to result from increasing uniaxial stresses (Figs. 2,4).

The volume incompressibility for the undeformed foil occurs during the glass transitions of 4:1 methanol-ethanol and paraffin oil. The beginning of the line broadening of ruby fluorescence in 4:1 methanol-ethanol and 1:1 pentaneisopentane at around 10 and $7 \mathrm{GPa}$, respectively, has previously been related to the glass transition in these liquids. ${ }^{1}$ The discontinuity in the compressibility is here interpreted to occur at the final solidification of these liquids. The saturation of the stresses (Figs. 2,6) and of the stacking faults (Fig. 4) in the region of the volume incompressibility suggests that the yield strength of the sample is exceeded in this pressure range. This is supported by the evidence for plastic deformation from the strong irreversible increase in the linewidths and in the Lorentzian character of the line profiles starting at the lower pressure limit of the volume incompressibility (Figs. 7,8). According to the von Mises yield criterion, the difference in principal stresses $t=\sigma_{3}-\sigma_{1}$ should be equal to the yield strength of the material as plastic deformation sets in. Because the calculation of $t$ from line shifts is affected by the presence of stacking faults, the maximum value of $t$ observed here is not identified with the yield strength (see also the discussion in part II).

The region of the volume incompressibility is here associated with work hardening during the glass transition of the pressure media. The continuing changes in the line profiles above the pressure of the volume discontinuity suggest further plastic deformation although there is no evidence for this from line shifts (the compressibility is isotropic in this range and similar to the one in the hydrostatic regime). The peak profiles are broadened both by elastic and plastic strains. Hence, the interpretation of any correlation (or lack thereof) between line profiles and stacking fault probability calculated from line shifts is difficult.

There is a correlation between volume incompressibility of sample and freezing of liquid pressure media only if the yield strength of the material is exceeded during the glass transition [compare the occurrence of the volume incompressibility in solid media: for $\mathrm{Cu}_{3} \mathrm{Au}$ in $\mathrm{NaCl}$ (Ref. 5) and for TaN in Pt (Ref. 30)]. The yield stress of the sample in the pressure range of the volume incompressibility can be estimated from known values at ambient conditions as follows. The critical resolved shear stress for $\langle\overline{1} 10\rangle\{111\}$ faults in ordered single crystals of $\mathrm{Cu}_{3} \mathrm{Au}$ in tension is about $20 \mathrm{MPa}$
$(=0.02 \mathrm{GPa}) .^{31}$ The tensile yield stress for polycrystals is obtained by multiplying this number by the Taylor factor for averaging over all possible orientations of the single crystal. ${ }^{32}$ With a Taylor factor of 3, valid for fec and bcc crystals, and equating tensile and compressive yield stresses, the minimum stress at the beginning of the volume incompressibility is estimated to be $0.06 \mathrm{GPa}$. The actual value is different from this because the compressive yield stress and tensile yield stress need not be equal (they differ by $\pm 10 \%$ in isostructural $\mathrm{Ni}_{3} \mathrm{Al}$ depending on the orientation and the slip direction). ${ }^{33}$ If it is assumed that pressure affects the yield stress only through its dependence on an effective elastic constant (here taken to be the shear modulus),${ }^{34}$ the yield stress would be increased to $0.08 \mathrm{GPa}$ at $5 \mathrm{GPa}$ and to 0.1 $\mathrm{GPa}$ at $10 \mathrm{GPa}$. The pressure dependence of the shear modulus was calculated with the equation presented by $\operatorname{Birch}^{35}$ which is based on Murnaghan's theory of finite strain. In general, one may also need to take into account a dependence of the yield stress on grain size. For disordered polycrystalline $\mathrm{Cu}_{3} \mathrm{Au}$ of 1 to $3 \mu \mathrm{m}$ grain size, the yield strength of 0.47 to $0.36 \mathrm{GPa}$ at ambient conditions ${ }^{36}$ would increase to 0.63 to $0.48 \mathrm{GPa}$ at $5 \mathrm{GPa}$ and to 0.78 to $0.6 \mathrm{GPa}$ at 10 $\mathrm{GPa}$. For comparison, the flow stress for Ni wafers deformed by shear under pressure ${ }^{37}$ was found to increase by a factor of $\approx 3$ at $4 \mathrm{GPa}$ and by $\approx 5$ at $10 \mathrm{GPa}$.

The shear stresses that can be transmitted by a liquid pressure medium depend on its viscosity. For the oil 1:1 pentaneisopentane, the viscosity at $4-5 \mathrm{GPa}$ is $10^{-6}-10^{-5} \mathrm{GPa} \mathrm{s}$ (using the relation $\eta=\mu \tau$ with $\eta$ the viscosity, $\mu$ is the shear modulus, and $\tau$ is the relaxation time). ${ }^{38,39}$ Based on an extrapolation ${ }^{1}$ of low-pressure data, ${ }^{39}$ the viscosity of methanol reaches this value around $7 \mathrm{GPa}$. This represents an increase of around $10^{7}-10^{8}$ over the value at ambient conditions for both 1:1 pentane-isopentane and methanol. The viscosity of the above media at the glass transition is around $10^{2} \mathrm{GPa} \mathrm{s} .{ }^{39,1}$ The relaxation times from which the viscosity data were calculated are between 2 and $76 \mathrm{~s}$ for $1: 1$ isopentane-pentane. ${ }^{39}$ The shear modulus of this oil is thus in the range $10^{-7}-10^{-8} \mathrm{GPa}$ at $4-5 \mathrm{GPa}$ and around $1 \mathrm{GPa}$ at the glass transition. These data support our conclusion that the deviation of the compression from an equation of state is caused by exceeding a critical stress characteristic of the material and its state (polycrystal versus single crystal, crystallite size of polycrystals).

The release curves for the Voigt fit parameters and the stress show that a large fraction of the accumulated strains do not relax and hence are plastic strains due to deformation. Most of the volume strains accumulated in the pressure range 7-12 GPa in 4:1 methanol-ethanol relax between 11 and 3 $\mathrm{GPa}$ (the hysteresis loop of the volume is almost closed). This pressure range brackets the pressure at which uniaxial stresses were first noticeable upon pressure increase (7 GPa). This suggests that the strain relaxation in the sample occurs when nonhydrostatic stresses in the pressure medium decrease below some critical value.

The initial deviation from an equation of state for $\mathrm{Cu}_{3} \mathrm{Au}$ in 4:1 methanol-ethanol occurs at pressures as low as $7 \mathrm{GPa}$, far below the glass transition which has hitherto been regarded as the hydrostatic limit. ${ }^{1}$ For $\mathrm{Cu}_{3} \mathrm{Au}$ in paraffin oil, plastic deformation was observed to set in around 4-5 GPa, 
below the freezing pressure of oil $(7 \mathrm{GPa})$ as determined from broadening of ruby fluorescence lines. ${ }^{1}$ This illustrates the necessity to define the hydrostatic limits of pressure media with respect to both the elastic properties of the sample and its state (single crystal versus polycrystal). In a polycrystal, there may be microstresses at grain boundaries due to the inability of neighboring crystals to accommodate changes in shape on compression.
${ }^{1}$ G. J. Piermarini, S. Block, and J. D. Barnett, J. Appl. Phys. 44, 5377 (1973).

${ }^{2}$ A. K. Singh and G. C. Kennedy, J. Appl. Phys. 45, 4686 (1974); 47, 3337 (1976); A. K. Singh, ibid. 73, 4278 (1993).

${ }^{3}$ M. A. Krivoglaz, X-ray and Neutron Diffraction in Nonideal Crystals (Springer-Verlag, Berlin, 1996).

${ }^{4}$ J. W. Otto, J. Appl. Crystallogr. (to be published).

${ }^{5}$ J. W. Otto, J. K. Vassiliou, and G. Frommeyer, J. Synchrotron Radiat. 4, 155 (1997).

${ }^{6}$ H. K. Mao and P. M. Bell, Carnegie Inst. Washington Yearb. 75, 824 (1976).

${ }^{7}$ R. A. Forman, G. J. Piermarini, J. D. Barnett, and S. Block, Science 176, 284 (1972).

${ }^{8}$ H. K. Mao, P. M. Bell, J. W. Shaner, and D. J. Steinberg, J. Appl. Phys. 49, 3276 (1978).

${ }^{9}$ J. W. Otto, Nucl. Instrum. Methods Phys. Res. A 384, 552 (1997).

${ }^{10}$ N. Funamori, T. Yagi, and T. Uchida, J. Appl. Phys. 75, 4327 (1994).

${ }^{11}$ E. Goens, J. Weert, and Stenzel, Z. Instrumentenk. 53, 242 (1933).

${ }^{12}$ R. Chiarodo, J. Green, I. L. Spain, and C. Bolsaitis, J. Phys. Chem. Solids 33, 1905 (1972).

${ }^{13}$ D. E. Mikkola and J. B. Cohen, J. Appl. Phys. 33, 892 (1962).

${ }^{14}$ D. E. Mikkola and J. B. Cohen, Acta Metall. 14, 105 (1966).

${ }^{15}$ S. B. Chakraborty and E. A. Starke, Jr., Acta Metall. 23, 63 (1975).

${ }^{16}$ A. Seeger and P. Haasen, Philos. Mag. 3, 470 (1958).

${ }^{17}$ M. A. Krivoglaz and K. P. Ryaboshapka, Fiz. Met. Metalloved. 15, 18 (1963).

${ }^{18}$ M. Wilkens, Acta Metall. 17, 1155 (1969).

${ }^{19}$ T. Ungár and A. Borbély, Appl. Phys. Lett. 69, 3173 (1996).

${ }^{20}$ B. E. Warren, X-ray Diffraction (Dover, New York, 1990).

${ }^{21}$ J. I. Langford, J. Appl. Crystallogr. 11, 10 (1978).
${ }^{22}$ H. P. Klug and L. R. Alexander, X-ray Diffraction Procedures for Polycrystalline and Amorphous Materials, 2nd ed. (Wiley, New York, 1974).

${ }^{23}$ J. I. Langford, R. Delhez, Th. H. de Keijser, and E. J. Mittemeijer, Aust. J. Phys. 41, 173 (1988).

${ }^{24}$ G. K. Williamson and W. H. Hall, Acta Metall. 1, 22 (1953).

${ }^{25}$ B. E. Warren and B. L. Averbach, J. Appl. Phys. 21, 595 (1950); 23, 497 (1952).

${ }^{26}$ Th. H. de Keijser, J. I. Langford, E. J. Mittemeijer, and A. B. P. Vogels, J. Appl. Crystallogr. 15, 308 (1982).

${ }^{27}$ A. R. Stokes and A. J. C. Wilson, Proc. Cambridge Philos. Soc. 38, 313 (1942); Proc. Phys. Soc. London 56, 283 (1944).

${ }^{28}$ D. Balzar and S. Popovic, J. Appl. Crystallogr. 29, 16 (1996).

${ }^{29}$ F. Birch, J. Geophys. Res. 83, 1257 (1978).

${ }^{30}$ J. K. Vassiliou, J. W. Otto, and R. F. Porter, in Proceedings of the XIV th AIRAPT/APS Conference on High-Pressure Science and Technology, edited by G. Schmidt, J. W. Shaner, M. Nicol, and M. Ross, AIP Conf. Proc. 309 (AIP, New York, 1994), pp. 461-464.

${ }^{31}$ D. Hull and D. J. Bacon, Introduction to Dislocations (Pergamon, Oxford, 1984).

${ }^{32}$ E. Kuramoto and D. P. Pope, Philos. Mag. 33, 675 (1976).

${ }^{33}$ Y. Umakoshi, D. P. Pope, and V. Vitek, Acta Metall. 32, 449 (1984).

${ }^{34}$ J. O. Chua and A. L. Ruoff, J. Appl. Phys. 46, 4659 (1975).

${ }^{35}$ F. Birch, J. Appl. Phys. 9, 279 (1938).

${ }^{36}$ S. M. L. Sastry, Mater. Sci. Eng. 22, 237 (1976).

${ }^{37}$ R. E. Riecker, L. C. Towle, and T. P. Rooney, Air Force Cambridge Res. Lab. Rept. 67-0475, 1967 (unpublished).

${ }^{38}$ P. W. Bridgman, Collected Experimental Papers (Harvard University Press, Cambridge, MA, 1964), Vol. VI, Paper No. 166, p. 3903.

${ }^{39}$ J. D. Barnett and C. D. Bosco, J. Appl. Phys. 40, 3666 (1964). 